\title{
NOVOS GENÓTIPOS DE TRITICUM DURUM L.: RENDIMENTO, ADAPTABILIDADE E QUALIDADE TECNOLÓGICA ${ }^{(1)}$
}

\author{
JOÃO CARLOS FELICIO ${ }^{(2)}$, CARLOS EDUARDO DE OLIVEIRA CAMARGO ${ }^{(2,8)}$, \\ CRISTINA DE PAULA DOS SANTOS MAGNO ${ }^{(3)}$, JOSÉ GUILHERME DE FREITAS ${ }^{(2,8)}$, \\ NELSON BORTOLETTO ${ }^{(4)}$, ARMANDO PETTINELLI JUNIOR ${ }^{(5)}$, PAULO BOLLER GALLO ${ }^{(6)}$ \\ \& JOSÉ CARLOS VILA NOVA ALVES PEREIRA ${ }^{(7)}$
}

\begin{abstract}
RESUMO
Compararam-se dezoito genótipos de Triticum durum L. e dois de Triticum aestivum L. em experimentos semeados em condições de irrigação por aspersão em diferentes regiões tritícolas paulistas, durante o período de 1995-97. Analisaram-se os seguintes parâmetros: rendimento de grãos, altura de plantas, ciclo da emergência à maturação, resistência às manchas foliares causadas por helmintosporiose, adaptabilidade, estabilidade, e qualidade tecnológica. O IAC 24 (T. aestivum) foi o mais produtivo, não diferindo dos genótipos de $T$. durum ALTAR/STN (12), MEMOS "S"/YAV79/3/SAPI"S"/TEAL"S"//HUI"S" (17), STN"S"/ 3/TEZ"S"/YAV79//HUI"S" (6), ALTAR/STN (11) e WIN"S"/SBA81A//STIL"S" (14) na comparação das médias para rendimento de grãos. Verificou-se que, em Ribeirão Preto, o rendimento médio de grãos foi de $5.332 \mathrm{~kg} /$ ha no período. Observaram-se valores semelhantes do coeficiente de determinação $\left(\mathrm{R}^{2}\right)$ para a maioria dos genótipos estudados, exceto para IAC 24 e IAC 1001. Este e o Anahuac apresentaram-se instáveis quanto à produção de grãos nos
\end{abstract}

\footnotetext{
(1) Recebido para publicação em 9 de junho de 1998 e aceito em 17 de fevereiro de 1999.

(2) Centro de Plantas Graníferas, Instituto Agronômico (IAC), Caixa Postal 28, 13001-970 Campinas (SP).

(3) Centro de Tecnologia de Chocolates, Balas, Confeitos e Panificação, Instituto de Tecnologia de Alimentos (ITAL), Caixa Postal 139, 13073-001 Campinas (SP).

(4) Núcleo de Agronomia do Noroeste, Votuporanga, IAC.

(5) Estação Experimental de Agronomia de Tatuí, IAC.

(6) Estação Experimental de Agronomia de Mococa, IAC.

(7) Núcleo de Agronomia da Alta Mogiana, Ribeirão Preto, IAC.

${ }^{(8)}$ Com bolsa de produtividade em pesquisa do CNPq.
} 
ambientes desfavoráveis e estável nos favoráveis, ao contrário do IAC 24. As infecções do agente causal de mancha-foliar foram baixas, sendo as ocorrências mais generalizadas em Tatuí. Os melhores genótipos de T. durum em relação à qualidade tecnológica foram os tratamentos IAC 1003 (1), SCO“S”/3/BD1814//BD708/BD1543/4/ROK"S” (13), ALTAR 84 (15) e MEMOS“S”/YAV79/3/SAPI"S"/TEAL"S"//HUI"S" (17), enquadrando-se os demais genótipos na classificação de farinha (sêmola) médio-fraca, produzindo massa de má qualidade. Estimou-se que as variáveis: tempo de desenvolvimento, sedimentação, índice de tolerância à mistura, energia de deformação da massa, número de queda, tenacidade versus elasticidade $(\mathrm{P} / \mathrm{L})$ e estabilidade seriam, em ordem de importância, parâmetros a considerar na seleção de cada genótipo, conjuntamente com o rendimento de grãos e sua adaptabilidade.

Termos de indexação: Triticum durum L., T. aestivum L., rendimento de grãos, doenças, adaptabilidade e qualidade tecnológica.

\section{ABSTRACT \\ NEW GENOTYPES OF TRITICUM DURUM L.: GRAIN YIELD, ADAPTABILITY AND TECHNOLOGICAL QUALITY}

Eighteen durum wheat and two bread wheat genotypes were evaluated for grain yield, plant height, cycle from emergence to maturity, resistance to leaf spots caused by Helminthosporium sp, adaptability, stability and technological quality, in experiments, under sprinkler irrigation, carried out at different regions of State of São Paulo, Brazil. The bread wheat IAC 24 presented the highest yield but not differing from the durum genotypes ALTAR/STN (12), MEMOS“S"/YAV79/3/SAPIS"S"/TEAL"S"//HUI"S” (17), STN"S"/3/ TEZ"S"/YAV79//HUI"S" (6), ALTAR/STN (11) and WIN"S”SBA81A//STIL"S”(14). At Ribeirão Preto site the grain yield mean of the evaluated genotypes was $5,332 \mathrm{~kg} / \mathrm{ha}$, in the period. Coefficients of determination for all genotypes were similar with exception for IAC 24 and IAC 1001. The last one, a durum wheat, showed instable in relation to grain yield under not favorable environments, and stable under favorable environments. The bread wheat IAC 24 presented opposite behaviour. The infection of the causal agent of leaf spot was low, but at Tatuí site the occurrence of this disease was more generalized. The best durum wheat genotypes in relation to quality were IAC 1003 (1), SCO"S"/3/BD1814//BD708/BD1543/4/ ROK"S"(13), ALTAR 84 (15) and MEMOS“S"/YAV79/3/SAPI"S"/TEAL"S"/HUI"S" (17). The other durum wheat genotypes presented medium to low flour strength producing bad quality dough. Dough development time, sedimentation test, index of mixture tolerance, energy for deformation of the dough, falling number, tenacity versus elasticity $(\mathrm{P} / \mathrm{L})$ and dough stability were in order of importance the parameters to be considered combined with grain yield and adaptability for the genotype selections.

Index terms: Triticum durum L., T. aestivum L., grain yield, diseases, adaptability, and technological quality.

\section{INTRODUÇÃO}

O trigo comum, pertencente à espécie Triticum aestivum L., constitui um alotetraplóide com três genomas básicos, A, B e D, cada um deles representado por sete pares de cromossomos. Os fatores genéticos responsáveis pela qualidade de panificação localizam-se nos cromossomos do genoma D. 
O Triticum durum L., denominado de trigo macarroneiro, tem somente os genomas A e B, não apresentando qualidades para a panificação (Carvalho, 1982).

O trigo comum utilizado para panificação cobre cerca de $90 \%$ da área semeada com trigo em todo o mundo e produz em volta de $94 \%$ da produção mundial de trigo. O T. durum, chamado também de trigo cristalino ou macarroneiro, abrange somente $9 \%$ da área trigueira mundial e representa $5 \%$ de toda a produção mundial (Hanson et al., 1982).

O rendimento médio do T. durum é mais baixo que o do T. aestivum, em vista da tendência que existe de cultivar o trigo duro em climas semi-áridos, pois considera-se que esta espécie está mais bem adaptada a regiões com precipitações pluviais escassas. Entretanto, em condições de irrigação, os cultivares modernos apresentam rendimentos similares aos dos melhores cultivares de trigo comum (Hanson et al., 1982).

Os cultivares de T. durum em cultivo apresentam grãos brancos e cristalinos (âmbar), mostrando-se passíveis de germinar na espiga quando maduros se submetidos à chuva no campo antes da colheita. Esse é um problema sério na implantação da cultura fora das regiões de clima semi-árido. São, ainda, sensíveis à toxicidade de $\mathrm{Al}^{3+}$ (Camargo et al., 1993).

Pelo número de queda, pode-se estimar a capacidade de fermentação que a massa de determinada farinha apresenta, pois trigos germinados ou em vias de germinar apresentam atividade amilásica elevada e liqüefazem a massa, dificultando o processo industrial (Mandarino, 1993).

As variedades de T.durum variam consideravelmente nas características reológicas da sêmola. No farinógrafo, variedades dessa espécie de glúten fraco apresentam um curto tempo de desenvolvimento da massa (TDM) e um alto índice de tolerância (IT), enquanto variedades de glúten forte geralmente têm um longo TDM e um muito baixo IT. O conteúdo de proteínas, entretanto, afeta esses parâmetros (Irvine et al., 1961).

Segundo Quaglia (1988), o alveograma de farinhas de $T$. durum indica alta tenacidade $(\mathrm{P})$ e baixa elasticidade (L), com a relação $\mathrm{P} / \mathrm{L}$ acima de 1,5 , e o índice de deformação da massa (W) igual ou maior que 200.

Este trabalho teve por objetivo avaliar dezoito genótipos de Triticum durum L. provenientes dos trabalhos de introdução e avaliação de trigo do Instituto Agronômico (Campinas, SP), em comparação com dois cultivares de trigo comum em condições de irrigação por aspersão em diferentes zonas tritícolas paulistas, quanto à produção de grãos, altura de plantas, ciclo da emergência à maturação e qualidade industrial da sêmola.

\section{MATERIAL E MÉTODOS}

Foram avaliados dezoitos genótipos de Triticum durum L., introduzidos do Centro Internacional de Melhoramento de Milho e Trigo (CIMMYT), México, juntamente com os genótipos Anahuac e IAC 24 (T. aestivum L.), durante o período 1995-97, em experimentos semeados em condição de irrigação por aspersão em diferentes regiões tritícolas do Estado, a saber: Tatuí (Zona D), Votuporanga (Zona F), Ribeirão Preto (Zona $\mathrm{G})$ e Mococa (Zona $\mathrm{H})$. São regiões tritícolas distintas quanto às condições climáticas e de fertilidade do solo, de acordo com as recomendações da Comissão Técnica de Trigo da Secretaria de Agricultura e Abastecimento para o Estado de São Paulo (Instituto Agronômico, 1996).

No Quadro 1 estão contidos os valores relativos à análise química da terra, nas profundidades de 0-20, 20-40 e 40-60 cm, dos diversos locais dos experimentos, segundo o método descrito por Raij \& Quaggio (1983).

Os ensaios foram delineados em blocos completos ao acaso, com quatro repetições por local. Cada parcela foi constituída por seis linhas de $3 \mathrm{~m}$ de comprimento, com espaçamentos de $0,20 \mathrm{~m}$ entre si, com separação lateral de 0,60 m entre as parcelas. Procedeu-se à semeadura com 80 sementes viáveis por metro linear de sulco, sendo a colheita realizada na área total das parcelas, ou seja, 3,6 $\mathrm{m}^{2}$.

A relação dos genótipos avaliados encontra-se no Quadro 2. 
A adubação mineral foi feita a lanço, com posterior incorporação ao solo antes da semeadura. A quantidade de fertilizantes aplicada nos diferentes locais basearam-se nas recomendações de Raij et al. (1996) e de acordo com os resultados das análises químicas do solo. Na adubação em cobertura, aplicaram-se $40 \mathrm{~kg} / \mathrm{ha}$ de $\mathrm{N}$ aos 20-30 dias após a emergência das plântulas.

Adotou-se, para a irrigação, o método proposto por Silva et al. (1984): consiste em uma irrigação inicial após a semeadura, de 40-60 mm, com a finalidade de umedecer o perfil do solo, bem como a instalação de tensiômetros em diferentes pontos, à profundidade de $12 \mathrm{~cm}$. Efetuaram-se as irrigações complementares quando a média das leituras dos tensiômetros indicava -0,06 MPa, determinando-se a lâmina líquida aplicada pela evaporação acumulada, medida no tanque classe A, entre os intervalos de irrigação.

Para avaliação do comportamento dos cultivares em relação às manchas-foliares, causadas principalmente por Helminthosporium sativum (mancha-marrom) e Helminthosporium repentis (crestamento amarelo), em condições naturais de infecção em campo, determinou o percentual de infecção nas folhas, adotando-se o método proposto por Metha (1978).

No decorrer do período experimental, foram obtidos os seguintes dados:

Ciclo da emergência à maturação: número de dias decorridos da emergência das plântulas até a maturação (colheita). Os genótipos com ciclo de 120-

Quadro 1. Análise química das amostras compostas dos solos dos locais dos experimentos dos genótipos de T. durum L. e T. aestivum L. avaliados em condição de irrigação por aspersão, referente a 1996

\begin{tabular}{|c|c|c|c|c|c|c|c|c|c|c|c|}
\hline Local & $\begin{array}{c}\text { Prof. } \\
\text { do solo }\end{array}$ & $\mathrm{P}$ & M.O. & $\begin{array}{c}\mathrm{pH}- \\
\mathrm{CaCl}_{2}\end{array}$ & $\mathrm{~K}$ & $\mathrm{Ca}$ & $\mathrm{Mg}$ & $\mathrm{H}+\mathrm{Al}$ & $\mathrm{S}$ & $\mathrm{T}$ & $\mathrm{V}$ \\
\hline & $\mathrm{cm}$ & $\mathrm{mg} \cdot \mathrm{dm}^{-3}$ & g. $\mathrm{kg}^{-1}$ & & 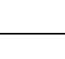 & 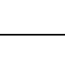 & $-\mathrm{mn}$ & $1_{c} \cdot \mathrm{dm}^{-3}$ & & 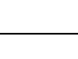 & $\%$ \\
\hline \multirow[t]{3}{*}{ Mococa ............... } & $0-20$ & 26 & 20 & 5,1 & 2,1 & 32 & 12 & 25 & 46 & 71 & 65 \\
\hline & $20-40$ & 3 & 16 & 4,6 & 1,2 & 16 & 7 & 31 & 24 & 55 & 44 \\
\hline & $40-60$ & 2 & 11 & 5,0 & 0,9 & 12 & 7 & 25 & 20 & 45 & 44 \\
\hline \multirow[t]{3}{*}{ Ribeirão Preto ... } & $0-20$ & 39 & 30 & 5,2 & 2,3 & 31 & 14 & 38 & 47 & 85 & 55 \\
\hline & $20-40$ & 17 & 16 & 5,1 & 1,2 & 40 & 14 & 38 & 55 & 93 & 59 \\
\hline & $40-60$ & 17 & 19 & 5,0 & 2,9 & 40 & 15 & 43 & 58 & 101 & 57 \\
\hline \multirow[t]{3}{*}{ Tatuí .......................... } & $0-20$ & 64 & 25 & 5,3 & 6,0 & 60 & 15 & 34 & 81 & 115 & 70 \\
\hline & $20-40$ & 29 & 23 & 4,7 & 3,8 & 41 & 13 & 52 & 58 & 110 & 53 \\
\hline & $40-60$ & 7 & 19 & 4,1 & 2,5 & 21 & 9 & 99 & 33 & 132 & 25 \\
\hline \multirow[t]{3}{*}{ Votuporanga ....... } & $0-20$ & 38 & 14 & 5,2 & 2,0 & 25 & 9 & 18 & 37 & 55 & 67 \\
\hline & $20-40$ & 26 & 14 & 5,1 & 2,1 & 23 & 8 & 18 & 33 & 51 & 65 \\
\hline & $40-60$ & 8 & 9 & 5,0 & 1,8 & 16 & 8 & 16 & 26 & 42 & 62 \\
\hline
\end{tabular}


$125,126-135$ e maiores que 135 dias foram considerados, respectivamente, de ciclo precoce, médio e tardio.

Altura das plantas: distância, em centímetros, do nível do solo ao ápice da espiga, com exclusão das aristas, medida no final do ciclo, estimando-se a média de diferentes pontos de cada parcela.

Rendimento de grãos: massa da produção total de cada parcela, a qual foi transformada para quilograma/hectare.
Para avaliação da qualidade tecnológica da sêmola, utilizaram-se os experimentos semeados nas Estações Experimentais de Tatuí e Votuporanga. Para isso, juntaram-se as produções de grão das quatros parcelas de cada experimento (local), a fim de haver quantidade de grãos suficiente para as seguintes determinações:

- umidade dos grãos: determinada pelo método no ${ }^{-44-15 A}$ da AACC (1983);

Quadro 2. Ciclo (da emergência à maturação) e altura média das plantas de genótipos de T. durum L. e T. aestivum L., avaliadas em condição de irrigação por aspersão nas localidades paulistas de Tatuí (Zona D), Ribeirão Preto (Zona G), Votuporanga (Zona F) e Mococa (Zona H) em 1995-97

\begin{tabular}{|c|c|c|}
\hline Genótipos & $\mathrm{Ciclo}^{(1)}$ & Altura \\
\hline & & $\mathrm{cm}$ \\
\hline 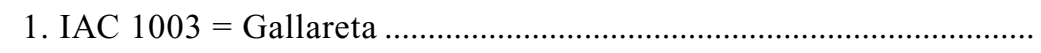 & Precoce & 60 \\
\hline 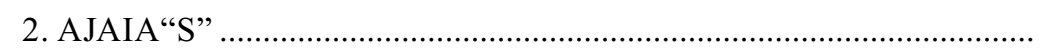 & Médio & 65 \\
\hline 3. IAC 1002=61150/LDS//GLL“S”/3/GR“S”/4/MEX“S”/5/S15/CR ... & Médio & 70 \\
\hline 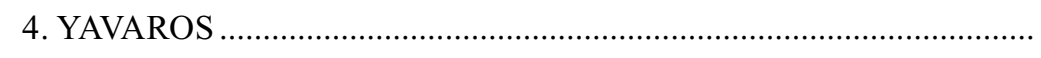 & Médio & 65 \\
\hline 5. STN"S"//HUI“S”/SOMOS “S” ........... & Médio & 70 \\
\hline 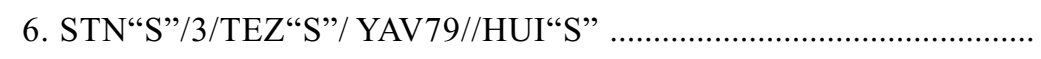 & Precoce & 80 \\
\hline 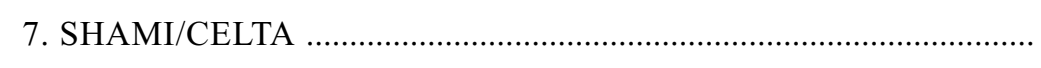 & Precoce & 75 \\
\hline 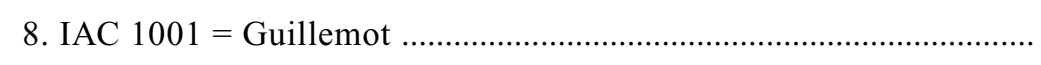 & Tardio & 75 \\
\hline 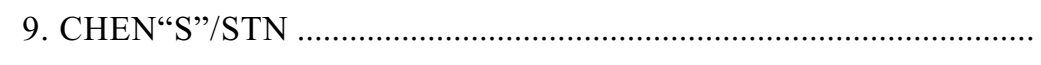 & Médio & 85 \\
\hline 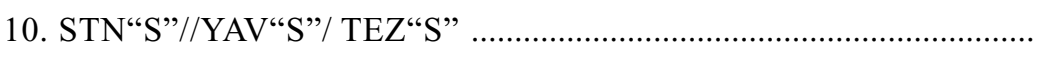 & Médio & 80 \\
\hline 11. ALTAR/STN/(A) & Médio & 70 \\
\hline 12. ALTAR/STN /(B) & Médio & 75 \\
\hline 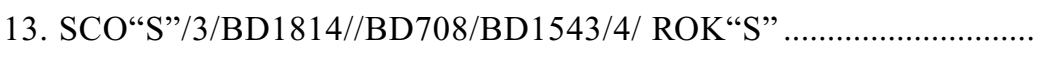 & Tardio & 70 \\
\hline 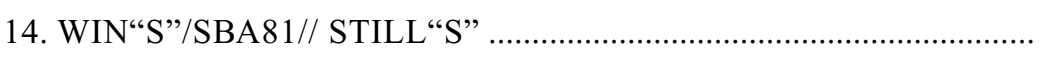 & Médio & 80 \\
\hline 15. ALTAR 84 . & Precoce & 60 \\
\hline 16. SULA "S" & Médio & 65 \\
\hline 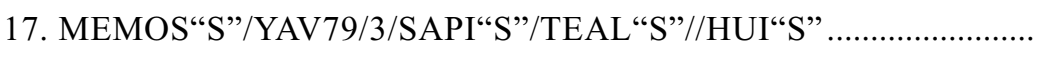 & Tardio & 70 \\
\hline 18. STN"S"/GOTE & Médio & 70 \\
\hline 19. ANAHUAC ${ }^{(2)}$ & Médio & 70 \\
\hline 20. IAC $24{ }^{(2)}$ & Médio & 75 \\
\hline
\end{tabular}

(1) Precoce de 120 a 125 dias da emergência à maturação; médio: entre 126 e 135 dias; tardio: > 135 dias.

(2) T. aestivum L. 
- rendimento de extração de farinha (sêmola): obtido pelo moinho experimental Brabender Quadrumat Senior, trabalhando com $2 \mathrm{~kg}$ de grãos, com base no método $\mathrm{n}^{\circ} 26-20$ da AACC (1983). Os grãos tiveram o teor de água ajustado para $15 \%$, em torno de 16-20 horas antes da moagem;

- teste de microssedimentação com dodecil sulfato de sódio (MS-SDS): pelo método modificado por Peña \& Amaya (1985);

- número de queda (falling number): determinado pelo método n. ${ }^{\circ}$ 56-81B da AACC (1983);

- propriedades de mistura da massa: determinadas no farinógrafo "Brabender", pelo sistema farinha-água, de acordo com o método n. ${ }^{\circ}$ 54-21 da AACC (1983). Os parâmetros usados para interpretar o farinógrafo foram absorção de água, tempo de desenvolvimento da massa (TDM), estabilidade (EST), índice de tolerância à mistura (ITM) e valor volumétrico (VV) em unidades farinográficas;

- propriedades de extensão da massa: determinadas com o equipamento Alveógrafo "Chopin", de acordo com o método no 54-30 da AACC (1983). Os parâmetros básicos das curvas alveográficas foram: pressão máxima $(\mathrm{P})$; abscissa média de ruptura $(\mathrm{L})$; índice de configuração da curva $(\mathrm{P} / \mathrm{L})$ e energia de deformação da massa (W).

O rendimento de grãos de cada genótipo foi submetido à análise estatística de acordo com o modelo para grupos de experimentos (Pimentel-Gomes, 1970), empregando-se, para a comparação das médias, o teste de Duncan a $5 \%$ de probabilidade.

Também procedeu-se à análise de estabilidade e adaptabilidade pelo método de Cruz et al. (1989), tendo como parâmetros de adaptabilidade a média e as respostas lineares aos ambientes desfavoráveis $\left(\mathrm{B}_{1}\right)$ e aos favoráveis $\left(\mathrm{B}_{1}+\mathrm{B}_{2}\right)$, sendo a estabilidade avaliada pelo desvio da regressão $\left(\mathrm{S}^{2} \mathrm{~d}\right)$.

Os dados médios anuais de rendimento de grãos, e de sêmola, MS-SDS, falling number, e os índices farinográficos absorção de água e estabilidade e os alveográficos $\mathrm{P} / \mathrm{L}$ e W médios obtidos, foram submetidos ao estudo de divergência genética por componentes principais e método de agrupamento de Tocher (Cruz \& Regazzi, 1994).

\section{RESULTADOS E DISCUSSÃO}

Os ciclos médios da emergência à maturação e as alturas médias das plantas dos genótipos, considerando a média dos experimentos, encontram-se no Quadro 2. Os genótipos 1, 6, 7 e 15 de T. durum são de ciclo precoce; 8, 13 e 17, de ciclo tardio, e os demais, de ciclo médio, em relação aos de trigo comum Anahuac e IAC 24. Os genótipos mostraram altura média variando de 60 a $80 \mathrm{~cm}$, enquadrando-se como porte semi-anão.

O rendimento médio de grãos e o resumo da análise da variância conjunta para cada local e para todos os experimentos avaliados em Tatuí (Zona D), Votuporanga (Zona F), Ribeirão Preto (Zona G) e Mococa (Zona H), em 1995-97, encontram-se no Quadro 3.

Os dados da análise conjunta envolvendo todos os experimentos mostraram efeitos significativos para anos (A), locais (L) e genótipos (G), bem como para as interações A x L, A x G, L x G e A x L x G. Todas as análises conjuntas individuais por local apresentaram efeitos significativos para anos. Para genótipos os efeitos foram significativos em Tatuí, Votuporanga e Ribeirão Preto e não significativo em Mococa, ocorrendo o mesmo com a interação ano x genótipos.

O genótipo de $T$. aestivum IAC 24 foi o mais produtivo em Tatuí e Votuporanga. Os genótipos de $T$. durum, exceto o 18, apresentaram rendimentos superiores aos genótipos IAC 24 e Anahuac em Ribeirão Preto. Explica-se esse fato provavelmente pela boa composição química do solo até a profundidade de $60 \mathrm{~cm}$ (Quadro 1), resultados que concordam com os obtidos por Camargo et al. (1993) e Hanson et al. (1982), os quais mostraram que cultivares de T. durum produzem altos rendimentos com irrigação em solos de alta fertilidade.

A análise de estabilidade pelo método bissegmentado proposta por Cruz et al. (1989), apresentando a vantagem de caracterizar cada genótipo quanto ao seu ambiente, encontra-se na Quadro 4. 
Quadro 3. Rendimento médio de grãos dos genótipos de T. durum L. e T. aestivum L. avaliados em condição de irrigação por aspersão nas localidades de Tatuí (Zona D), em Ribeirão Preto (Zona G), Votuporanga (Zona F) e Mococa (Zona H) no Estado de São Paulo em 1995-97

Genótipo Tatuí Votuporanga $\begin{gathered}\text { Ribeirão } \\ \text { Preto }\end{gathered}$ Mococa Médias ${ }^{(1)}$

\begin{tabular}{|c|c|c|c|c|c|}
\hline \multirow[b]{2}{*}{ 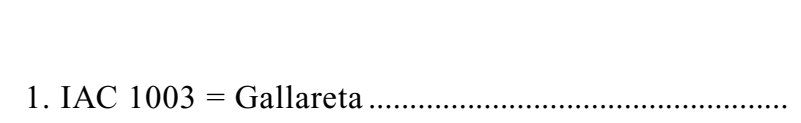 } & \multirow[b]{2}{*}{$2.915 \mathrm{c}-\mathrm{e}$} & \multirow[b]{2}{*}{$2.223 \mathrm{c}-\mathrm{f}$} & $\mathrm{kg} \cdot \mathrm{ha}^{-1}$ & & \\
\hline & & & $5.227 \mathrm{~b}-\mathrm{e}$ & 3.967 & $3.583 \mathrm{e}-\mathrm{g}$ \\
\hline 2. AJAIA “S" ...................... & $3.004 \mathrm{c}-\mathrm{e}$ & 2.038 ef & $5.360 \mathrm{a}-\mathrm{d}$ & 3.963 & $3.591 \mathrm{e}-\mathrm{g}$ \\
\hline 3. IAC 1002 & $3.017 \mathrm{c}-\mathrm{e}$ & $2.276 \mathrm{c}-\mathrm{e}$ & $5.593 \mathrm{ab}$ & 4.113 & $3.750 \mathrm{~b}-\mathrm{e}$ \\
\hline 4. YAVAROS . & $3.062 \mathrm{c}-\mathrm{e}$ & $2.243 \mathrm{c}-\mathrm{e}$ & $5.486 \mathrm{a}-\mathrm{c}$ & 3.875 & $3.667 \mathrm{de}$ \\
\hline 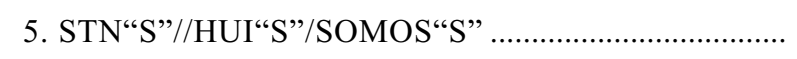 & $3.069 \mathrm{c}-\mathrm{e}$ & $2.055 \mathrm{ef}$ & $5.561 \mathrm{ab}$ & 3.773 & $3.615 \mathrm{~d}-\mathrm{f}$ \\
\hline 6. STN“S”/3/TEZ“S”/YAV79//HUI“S” .............................. & $3.664 \mathrm{~b}$ & $2.227 \mathrm{c}-\mathrm{f}$ & $5.433 \mathrm{a}-\mathrm{d}$ & 4.194 & $3.879 \mathrm{ab}$ \\
\hline 7. SHAMI/CELTA & $2.965 \mathrm{c}-\mathrm{e}$ & $2.152 \mathrm{~d}-\mathrm{f}$ & $5.321 \mathrm{a}-\mathrm{d}$ & 3.886 & $3.581 \mathrm{e}-\mathrm{g}$ \\
\hline 8. IAC 1001 = Guillemot & $3.046 \mathrm{c}-\mathrm{e}$ & $1.850 \mathrm{f}$ & $5.404 \mathrm{a}-\mathrm{d}$ & 4.254 & $3.639 \mathrm{~d}-\mathrm{f}$ \\
\hline 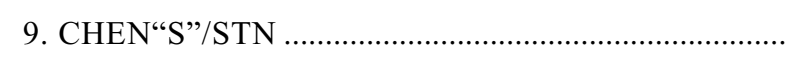 & $3.227 \mathrm{~cd}$ & $2.349 \mathrm{~b}-\mathrm{e}$ & $5.241 \mathrm{~b}-\mathrm{e}$ & 3.993 & $3.702 \mathrm{~b}-\mathrm{e}$ \\
\hline 10. STN"S"//YAV“S”/ TEZ“S”. & $3.144 \mathrm{~cd}$ & $2.272 \mathrm{c}-\mathrm{e}$ & $5.602 \mathrm{ab}$ & 3.942 & $3.740 \mathrm{~b}-\mathrm{e}$ \\
\hline 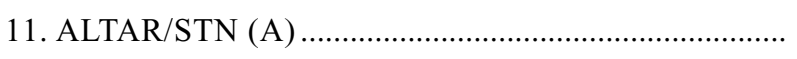 & $3.298 \mathrm{c}$ & $2.294 \mathrm{c}-\mathrm{e}$ & $5.681 \mathrm{a}$ & 4.173 & $3.861 \mathrm{a}-\mathrm{c}$ \\
\hline 12. ALTAR/STN (B) & $3.744 \mathrm{~b}$ & $2.243 \mathrm{c}-\mathrm{e}$ & $5.669 \mathrm{a}$ & 4.136 & $3.948 \mathrm{a}$ \\
\hline 13. SCO"S"/3/BD1814//BD708/BD1543/4/ ROK“S” .... & $3.094 \mathrm{~cd}$ & 2.083 ef & $5.574 \mathrm{ab}$ & 3.981 & $3.683 \mathrm{c}-\mathrm{e}$ \\
\hline 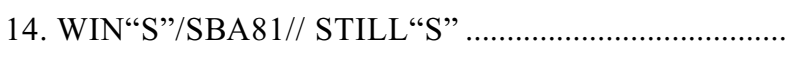 & $3.118 \mathrm{~cd}$ & $2.561 \mathrm{a}-\mathrm{c}$ & $5.315 \mathrm{a}-\mathrm{d}$ & 4.210 & $3.801 \mathrm{a}-\mathrm{d}$ \\
\hline 15. ALTAR 84 & $2.679 \mathrm{e}$ & $2.215 \mathrm{c}-\mathrm{f}$ & $5.065 \mathrm{~d}-\mathrm{f}$ & 3.673 & $3.408 \mathrm{~g}$ \\
\hline 16. SULA "S" & $2.946 \mathrm{c}-\mathrm{e}$ & $2.354 \mathrm{~b}-\mathrm{e}$ & $5.133 \mathrm{c}-\mathrm{f}$ & 3.991 & 3.606 ef \\
\hline 17. MEMOS“S”/YAV79/3/SAPI“S"/TEAL"S"//HUI"S” & $3.284 \mathrm{c}$ & $2.501 \mathrm{a}-\mathrm{d}$ & $5.545 \mathrm{ab}$ & 4.437 & $3.942 \mathrm{a}$ \\
\hline 18. STN"S"/GOTE & $2.962 \mathrm{c}-\mathrm{e}$ & $2.289 \mathrm{c}-\mathrm{e}$ & $4.926 \mathrm{e}-\mathrm{g}$ & 3.687 & $3.466 \mathrm{fg}$ \\
\hline 19. ANAHUAC ${ }^{(2)}$ & $2.854 \mathrm{de}$ & $2.668 \mathrm{ab}$ & $4.675 \mathrm{~g}$ & 3.620 & $3.454 \mathrm{fg}$ \\
\hline 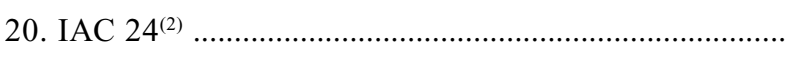 & $4.132 \mathrm{a}$ & $2.744 \mathrm{a}$ & $4.838 \mathrm{fg}$ & 4.083 & $3.949 \mathrm{a}$ \\
\hline Médias ............. & $3.161 \mathrm{C}$ & $2.262 \mathrm{D}$ & $5.332 \mathrm{~A}$ & $3.998 \mathrm{~B}$ & \\
\hline $\mathrm{F}$ (ano) & $396,86 * *$ & $72,56 * *$ & $156,46^{* *}$ & $16,57 * *$ & $133,89^{* *}$ \\
\hline $\mathrm{F}(\mathrm{local})$ & - & - & - & - & $753,23 * *$ \\
\hline 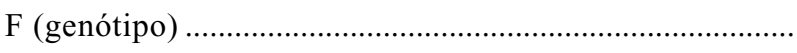 & $5,17 * *$ & $4,91 * *$ & $2,85 * *$ & $1,53 \mathrm{~ns}$. & $7,31 * *$ \\
\hline F (ano x local) & - & - & - & - & $15,39 * *$ \\
\hline $\mathrm{F}$ (ano x genótipo) & $2,40 * *$ & $2,87 * *$ & $1,44 * *$ & $0,88 \mathrm{~ns}$ & $2,98 * *$ \\
\hline F (local x genótipo) & - & - & - & - & $3,97 * *$ \\
\hline F (ano x local x genótipos) & - & - & - & - & $2,29 * *$ \\
\hline $\mathrm{CV}(\%)$ & 16,27 & 14,60 & 10,96 & 14,70 & 11,42 \\
\hline
\end{tabular}

(1) Médias para comparação da produtividade de grãos entre cultivares dentro de local e na média geral em letras minúsculas, e médias para comparação entre locais em letras maiúsculas. Médias seguidas por letras distintas diferem entre si pelo teste de Duncan a 5\%.

(2) T. aestivum L. * Significativo a 5\%; ns: Não significativo. 


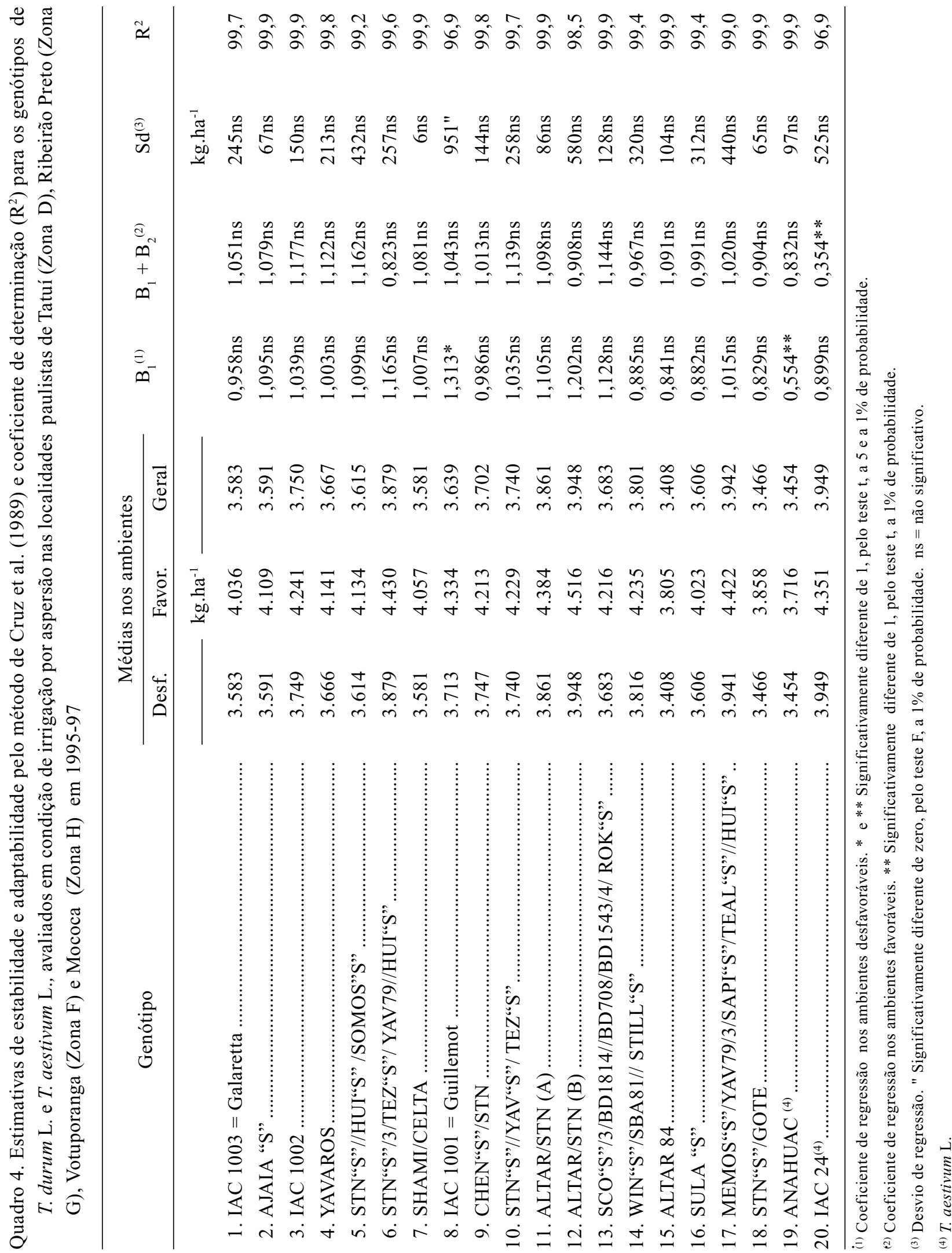


Em Ribeirão Preto (ambiente favorável), verificou-se rendimento médio de grão de $5.332 \mathrm{~kg} / \mathrm{ha}$ no período, contra $2.262 \mathrm{~kg} / \mathrm{ha}$ em Votuporanga (ambiente desfavorável), o menor rendimento médio (Quadro 3). A variação média de rendimento entre os ambientes foi maior do que se esperava, levando-se em consideração que os ambientes se situam em regiões semelhantes entre si, com exceção de Tatuí, que se localiza nos limites da aptidão ecológica para a cultura do trigo para o Estado de São Paulo em condições de sequeiro (Camargo et al., 1971).
Observam-se valores semelhantes do coeficiente de determinação $\left(\mathrm{R}^{2}\right)$ para a maioria dos genótipos $(98,5-99,9)$, exceto para IAC 1001 e IAC 24, com 96,9. Os genótipos IAC 1001 e Anahuac apresentaram-se instáveis nos ambientes desfavoráveis e estáveis nos favoráveis. O IAC 24 mostrou-se, estável em ambientes desfavoráveis e instável nos favoráveis.

No período avaliado, não ocorreram condições naturais favoráveis para altas infecções do agente causal de helmintosporiose. As mais generalizadas foram em Tatuí (Quadro 5), climaticamente mais favorável

Quadro 5. Ocorrência de helmintosporiose (B. sorokiniana) nos genótipos de T. durum L. e T. aestivum L., avaliados em condição de irrigação por aspersão nas localidades paulistas de Tatuí (Zona D), Ribeirão Preto (Zona G), Votuporanga (Zona F) e Mococa (Zona H) em1995-97

\begin{tabular}{|c|c|c|c|c|}
\hline Genótipo & Tatuí & $\begin{array}{c}\text { Votu- } \\
\text { poranga }\end{array}$ & $\begin{array}{c}\text { Ribeirão } \\
\text { Preto }\end{array}$ & Mococa \\
\hline & & $\%$ & & \\
\hline 1. IAC $1003=$ Galaretta & 35 & 5 & 5 & 1 \\
\hline 2. AJAIA “S" & 15 & 5 & 5 & 1 \\
\hline 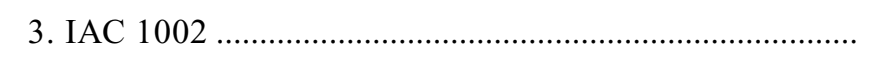 & 35 & 5 & 5 & 1 \\
\hline 4. YAVAROS & 15 & 5 & 5 & 1 \\
\hline 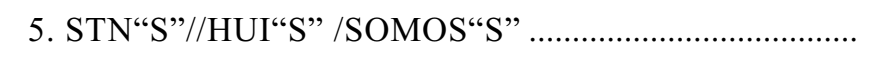 & 20 & 5 & 5 & 1 \\
\hline 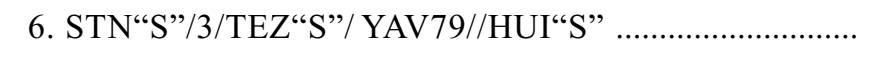 & 15 & 5 & 5 & 1 \\
\hline 7. SHAMI/CELTA & 20 & 5 & 5 & 1 \\
\hline 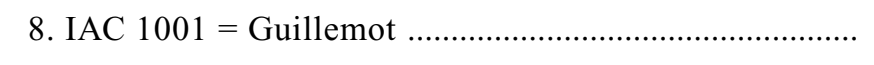 & 15 & 5 & 5 & 1 \\
\hline 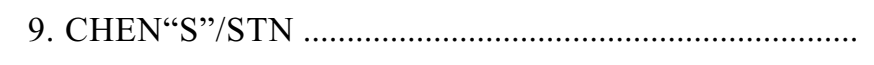 & 15 & 5 & 5 & 1 \\
\hline 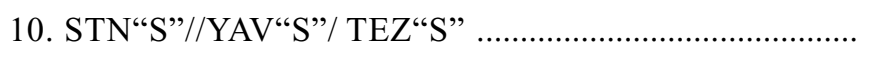 & 20 & 5 & 5 & 1 \\
\hline 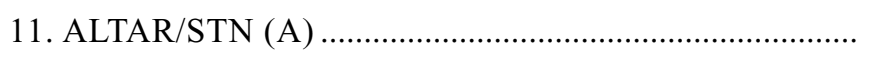 & 20 & 5 & 3 & 1 \\
\hline 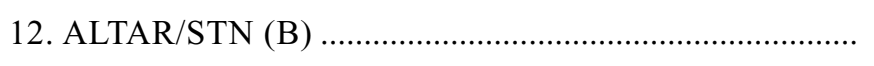 & 20 & 5 & 3 & 1 \\
\hline 13. SCO“S”/3/BD1814//BD708/BD1543/4/ ROK“S” ........ & 15 & 5 & 10 & 1 \\
\hline 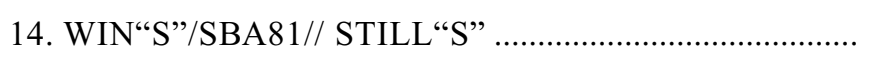 & 30 & 5 & 5 & 1 \\
\hline 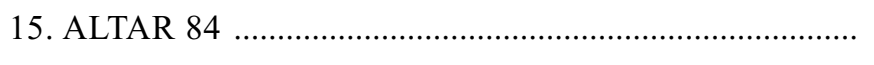 & 20 & 5 & 5 & 1 \\
\hline 16. SULA “S" & 30 & 5 & 5 & 1 \\
\hline 17. MEMOS“S”/YAV79/3/SAPI"S"/TEAL“S”//HUI“S”... & 10 & 5 & 5 & 1 \\
\hline 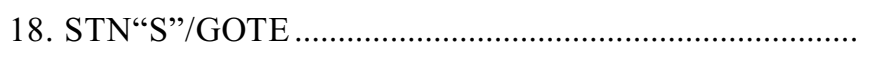 & 20 & 5 & 5 & 1 \\
\hline 19. ANAHUAC ${ }^{(1)}$ & 40 & 7,5 & 10 & 5 \\
\hline 20. IAC $24^{(1)}$ & 30 & 7,5 & 10 & 5 \\
\hline
\end{tabular}

(1) T. aestivum $\mathrm{L}$. 
à doença em razão da influência da umidade (Luz, 1982) por se localizar em região limítrofe àquela apta para a cultura no Sul do Estado (Camargo et al., 1971). Altas incidências de helmintosporiose podem afetar os grãos, provocando a doença denominada ponta-preta, que altera sensivelmente a qualidade do produto industrializado (sêmola), de acordo com Finney et al. (1987).
Os dados relativos ao teste de microssedimentação com sulfato dodecil de sódio (MS-SDS), o qual se baseia na propriedade de floculação das proteínas que formam o glúten, é altamente correlacionado com o conteúdo e com a qualidade das proteínas, para um mesmo cultivar - Quadro 6. Esse teste é empregado para avaliação do potencial de panificação (força do glúten) em programas de melhoramento.

Quadro 6. Microssedimentação com dodecil sulfato de sódio (MS-SDS), extração experimental de sêmola (EXT), número de queda (NQ) e características farinógraficas [tempo de desenvolvimento (TD), estabilidade e índice de tolerância à mistura (ITM)] e alveográficas [índice de configuração da curva $(\mathrm{P} / \mathrm{L})$ e energia de deformação da massa (W)] dos genótipos de T. durum L. e T. aestivum L. avaliados em condição de irrigação por aspersão nas localidades paulistas de Tatuí (Zona D), Ribeirão Preto (Zona G), Votuporanga (Zona F) e Mococa (Zona H) em 1995-97

\begin{tabular}{|c|c|c|c|c|c|c|c|c|}
\hline \multirow{2}{*}{ Genótipo } & \multirow{2}{*}{ MS-SDS } & \multirow{2}{*}{ EXT } & \multirow{2}{*}{ NQ } & \multicolumn{3}{|c|}{ Farinografia } & \multicolumn{2}{|c|}{ Alveografia } \\
\hline & & & & $\mathrm{TD}$ & EST & ITM & $\mathrm{P} / \mathrm{L}^{(1)}$ & $\mathrm{W}$ \\
\hline & $\mathrm{ml}$ & $\%$ & s & $\min$ & $\min$ & $\mathrm{UF}^{(2)}$ & & $10^{-4} \mathrm{j}$ \\
\hline 1. IAC $1003=$ Galaretta. & 29,2 & 48,5 & 402 & 5,0 & 6,0 & 45 & 1,30 & 200 \\
\hline 2. AJAIA "S" .. & 31,5 & 55,1 & 445 & 5,0 & 5,0 & 50 & 0,98 & 185 \\
\hline 3. IAC $1002 \ldots .$. & 25,8 & 63,5 & 375 & 2,5 & 2,0 & 85 & 0,53 & 100 \\
\hline 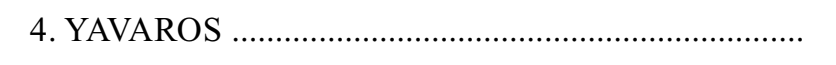 & 35,2 & 46,3 & 389 & 5,0 & 6,0 & 60 & 1,77 & 180 \\
\hline 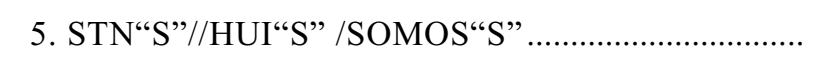 & 30,2 & 48,8 & 422 & 4,0 & 5,0 & 70 & 1,23 & 350 \\
\hline 6. STN"S"/3/TEZ"S"/ YAV79//HUI“S”.. & & 50,2 & 431 & 4,0 & 5,0 & 60 & 80 & 180 \\
\hline 7. SHAMI/CELTA …............................... & 23,8 & 44,8 & 386 & 3,0 & 3,0 & 80 & 1,29 & 155 \\
\hline 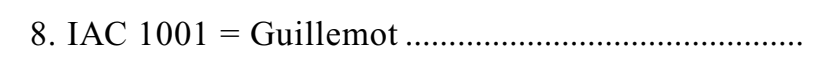 & 24,2 & 48,2 & 384 & 3,0 & 3,0 & 90 & 0,77 & 175 \\
\hline 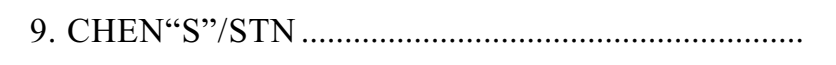 & 26,3 & 44,7 & 354 & 4,5 & 5,0 & 70 & 1,21 & 210 \\
\hline 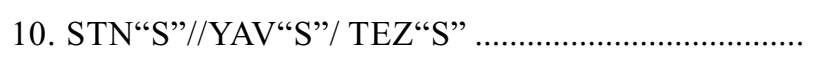 & 26,8 & 38,9 & 274 & 3,5 & 3,0 & 75 & 0,55 & 170 \\
\hline 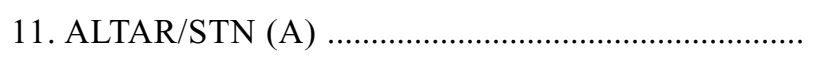 & 20,3 & 52,3 & 378 & 2,5 & 2,0 & 90 & 0,60 & 125 \\
\hline 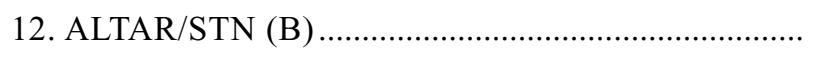 & 21,0 & 49,1 & 391 & 2,5 & 2,0 & 100 & 0,84 & 105 \\
\hline 13. SCO“S”/3/BD1814//BD708/BD1543/4/ ROK“S”. & 28,8 & 50,2 & 383 & 5,0 & 6,0 & 50 & 1,56 & 200 \\
\hline 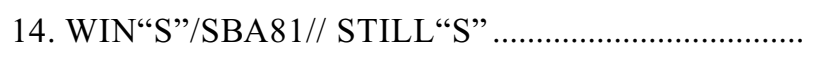 & 19,3 & 44,9 & 392 & 2,0 & 3,0 & 60 & 0,75 & 85 \\
\hline 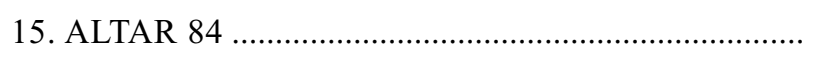 & 21,8 & 42,9 & 394 & 4,0 & 4,5 & 65 & 1,21 & 250 \\
\hline 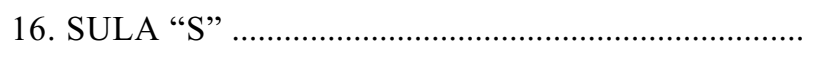 & 28,4 & 40,0 & 416 & 4,0 & 5,5 & 70 & 1,05 & 240 \\
\hline 17. MEMOS“S”/YAV79/3/SAPI“S”/TEAL“S”//HUI“S” & " 30,0 & 40,6 & 390 & 4,5 & 5,7 & 50 & 1,25 & 230 \\
\hline 18. STN“S”/GOTE ……………………… & 38,1 & 43,3 & 417 & 2,5 & 6,0 & 50 & 4,24 & 190 \\
\hline 19. ANAHUAC ${ }^{(3)}$ & 51,5 & 61,2 & 345 & 13,0 & 13,0 & 40 & 0,66 & 270 \\
\hline 20. IAC $24^{(3)} \ldots \ldots . .$. & 51,2 & 63,9 & 371 & 8,0 & 7,8 & 40 & 0,60 & 250 \\
\hline
\end{tabular}

(1) Índice de configuração da curva. ${ }^{(2)}$ Unidades farinográficas. ${ }^{(3)}$ T. aestivum L. 
Observa-se que os genótipos de T. aestivum apresentaram elevada força de glúten em comparação aos T. durum. Entre estes, destacaram-se os genótipos $1,2,4,5,6,17$ e 18 . O genótipo 14 revelou-se de baixa qualidade e os demais, de qualidade aceitável.

Quanto à extração experimental de farinha ou sêmola, os genótipos mostraram baixos índices, sobressaindo aqueles dos trigos comuns IAC 24 e Anahuac, e o genótipo 3 ( $T$. durum). O reduzido índice de extração apresentado pela maioria dos genótipos de $T$. durum, deve-se, provavelmente, ao tipo experimental de moagem apropriada para a extração de farinha de trigos comuns.

A atividade enzimática das farinhas foi avaliada pelo teste de falling number (número de queda). Valores inferiores a $150 \mathrm{~s}$ e os superiores a $300 \mathrm{~s}$ representam, respectivamente, alta e baixa atividade amilásica. Os genótipos, com exceção de 10 (T. durum) apresentaram baixa atividade amilásica (Quadro 6).

Os testes de farinógrafo e alveógrafo - Quadro 6 classificam os genótipos em grupos quanto à força do glúten, indicando as massas ou pastas que suportam melhor as condições de cocção. Os genótipos de T. durum 1, 13, 15 e 17, que possuem glúten forte e boas características industriais, apresentaram dados reológicos, como tempo de desenvolvimento, estabilidade e índice de tolerância à mistura (farinógrafo), e P/L e a energia de deformação da massa (W) no alveógrafo, de acordo com Dexter \& Matsuo (1980); outros genótipos também poderiam fazer parte deste grupo, mas tiveram o ITM muito alto. Os demais genótipos enquadraram-se na classificação de farinha (sêmola) médio-fraca, produzindo massas de má qualidade, que aderem durante o processo de cocção, apresentando a água de cocção uma coloração esbranquiçada em vista da presença de amido em suspensão (Mandarino, 1987).

A análise dos grupos similares para o caráter da qualidade industrial dos genótipos, e com base no método proposto por Tocher, citado por Cruz \& Regazzi (1994), permitiu o estabelecimento dos seguintes grupos homogêneos: Grupo I: 1,13, 4, 17, 15,
$16,6,9,7,10,8,14,11,12,2$ e 5; Grupo II: 19 e 20; Grupo III: 4, e Grupo IV: 18. Observa-se que os genótipos de trigo comum se enquadraram no mesmo grupo e aqueles de $T$. durum formaram três grupos, sendo o I constituído por maior número de genótipos similares entre si.

Considerando a importância dos caracteres avaliados, para qualidade industrial sobre a variação total disponível entre os genótipos, e utilizando a técnica dos componentes principais, foi possível estimar que as variáveis TD, MS-SDS, ITM, W, NQ, P/L e EST (Quadro 6) devem, em ordem de importância, ser consideradas na seleção do genótipo; a extração experimental de farinha (sêmola) pouco contribuiu para o caráter de cada genótipo.

\section{CONCLUSÕES}

1. O genótipo de T. aestivum IAC 24 foi o mais produtivo em ambientes como os de Tatuí e Votuporanga. Em Ribeirão Preto, os genótipos de $T$. durum expressaram melhor performance que os trigos comuns.

2. Os genótipos IAC 1001 e Anahuac apresentaram-se instáveis nos ambientes desfavoráveis e estáveis nos favoráveis.

3. Os genótipos de T. durum 1, 13, 15 e 17 revelaram adequados valores reológicos para uso na fabricação de massas ou pastas industriais.

4. As variáveis tempo de desenvolvimento (TD), microssedimentação (MS-SDS), índice de tolerância à mistura (ITM), energia de deformação da massa (W), número de queda (falling number) (NQ), índice de configuração da curva $(\mathrm{P} / \mathrm{L})$ e estabilidade (EST) devem, em ordem de importância, ser consideradas na seleção do genótipo, conjuntamente com o rendimento de grãos e sua adaptabilidade.

5. A cultura de T. durum constitui alternativa para a agricultura no inverno sob irrigação por aspersão. 


\section{REFERÊNCIAS BIBLIOGRAFICAS}

\section{AMERICAN ASSOCIATION OF CEREAL CHEMISTS} (AACC). Approved methods of the American Association of Cereal Chemists. 8. ed. St. Paul, AACC, 1983. v.1.

CAMARGO, A.P. de. Aptidão climática para as culturas da soja, girassol e amendoim no Estado de São Paulo. In: Zoneamento da aptidão ecológica para a cultura da soja, girasssol e amendoim no Estado de São Paulo. São Paulo, Instióleos/Secretaria da Agricultura, 1971. p.2-28.

CAMARGO, C.E.O.; FELICIO, J.C.; FERREIRA FILHO, A.W.P.; BARROS, B.C.; PETTINELLI JÚNIOR, A.B. \& SANTOS, R. R. dos. Trigo duro, trigo comum e triticale: avaliação de linhagens em condições de irrigação e de solução nutritiva. Arquivos do Instituto Biológico, São Paulo, 60(1/2):38-47, 1993.

CARVALHO, F. I. F. de. Genética quantitativa. In: Trigo no Brasil. Campinas, Fundação Cargill, 1982. v.1, p 63-94.

CRUZ, C.D. \& REGAZZI, A. J. Modelos biométricos aplicados ao melhoramento genético. Viçosa, Imprensa Universitária, 1994. 390p.

CRUZ, C.D.; TORRES, R.A. \& VENCOVSKY, R. An alternative approach to the stability analysi proposed by Silva \& Barreto. Revista Brasileira de Genética, Ribeirão Preto, 12:567-580, 1989.

DEXTER, J.E. \& MATSUO, R. R. Relationship between durum wheat protein properties and pasta dough rheology and spaghetti cooking quality. Journal of Agricultural and Food Chemistry, Washington, 28:899-902, 1980.

FINNEY, K.F.; YAMAZAKI, W.T.; YOUNGS, V.L. \& RUBENTHALER, G.L. Quality of hard, soft, and durum wheats. In: HEYNE. E.G., ed. Wheat and Wheat Improvement. 2.ed. Madison, 1987. p.677-748.

HANSON, H.; BORLAUG, N.E. \& ANDERSON, R.G. Trigo en el tercer mundo. México, Centro Internacional de Mejoramiento de Maiz y Trigo, 1982. 166p.

INSTITUTO AGRONÔMICO. Reunião da Comissão Técnica de Trigo, da Secretaria de Agricultura e Abastecimento do Estado de São Paulo: recomendações para 1996. Campinas, 1996. 68p. (Boletim técnico, 167)
IRVINE, G. N.; BRADLEY, J.W. \& MARTINI, G.C. A farinograph technique for macaroni doughs. Cereal Chemistry, St.Paul, 38:153, 1961.

LUZ, W.C. da. Influência do período de umidificação pósinoculação na reação de cultivares de trigo à mancha foliar (Cochiliobolus sativus). In REUNIÃO NACIONAL DE PESQUISA DE TRIGO, 12., Cascavel, 1982. Anais. Passo Fundo, EMBRAPA/CNPT, 1982. p186 -191.

MANDARINO, J.M.G. Aspectos importantes para a qualidade do trigo. Londrina, EMBRAPA-CNPSo, 1993. 32p. (EMBRAPA-CNPSo, Documento, 60)

METHA, Y.R. Doenças do trigo e seu controle. São Paulo, Agronômica Ceres, 1978. 190p. (Ceres, 20)

QUAGLIA, G.B. Other durum wheat products. In: FABRIANI, G. \& LINTAS, G., eds. Durum wheat chemistry and technology. St. Paul, 1988. v.1, p.263-282.

PEÑA,R.J. \& AMAYA, A. Evaluación rápida de la fuerza de gluten en trigos harineros, trigos cristalinos y triticales com la prueba de sedimentación con dodecil sulfato de sodio. México, Centro Internacional de Mejoramiento de Maiz y Trigo (CIMMYT), 1985. 6p (Mimeografado)

PIMENTEL-GOMES, F. Curso de estatistica experimental. 4. ed. rev. ampl. Piracicaba, Nobel, 1970. 430p.

RAIJ, B. van; CANTARELLA, H; QUAGGIO, J.A. \& FURLANI, A. M. C. Recomendações de adubação e calagem para o Estado de São Paulo. Campinas, Instituto Agronômico, Fundação IAC, 1996. 285p. (Boletim técnico, 100)

RAIJ, B. van \& QUAGGIO, J.A. Métodos de análises do solo para fins de fertilidade. Campinas, Instituto Agronômico, 1983. 31 p. (Boletim técnico, 81)

SILVA, E.M.; LUCHIARI JUNIOR, A.; GUERRA, A.F. \& GOMIDE, R.L. Recomendações sobre o manejo de irrigação em trigo para a região dos cerrados. In: REUNIÃO DA COMISSÃO NORTE BRASILEIRA DE PESQUISA DE TRIGO, 10., Campinas, 1984. Anais. Brasília, EMBRAPA-CPAC, 1984. 60p. 